\title{
Recherche de forme des gilets de sauvetage gonflables
}

\author{
Julien Troufflard ${ }^{\mathrm{a}}$, Jean-Marc Cadou et Gérard Rio \\ Laboratoire de Génie Mécanique et Matériaux, Université de Bretagne Sud, Rue de Saint Maudé, BP 92116, \\ 56321 Lorient Cedex, France
}

Reçu le 28 février 2008, accepté le 31 octobre 2008

\begin{abstract}
Résumé - Cet article présente une application de la recherche de forme aux gilets de sauvetage gonflables. La recherche de forme est le nom générique désignant le processus de conception de la forme globale des structures légères instables telles que les structures gonflables. L'état d'équilibre statique des gilets gonflables est recherché pour analyser leur forme, le volume contenu et la localisation des zones de plis. La méthode de relaxation dynamique avec amortissement cinétique permet d'éviter le problème de singularité de la matrice de raideur et le problème d'instabilité locale dans les zones de plis. Sa rapidité de convergence est très dépendante de la formulation de la matrice des masses. Dans cet article, plusieurs expressions des masses sont testées et leurs performances comparées entre elles sur un cas de gilet de sauvetage gonflable.
\end{abstract}

Mots clés : Recherche de forme / relaxation dynamique / étude de convergence / gilet de sauvetage gonflable

\begin{abstract}
Form finding of inflatable lifejackets. This paper presents an application of form-finding for lifejackets. Form-finding is the process in which the design of lightweight structures as inflatable structures is researched. The static equilibrium state of lifejackets is required to analyse their shape, contained volume and the wrinkling zones localisation. The dynamic relaxation method with kinetic damping avoids the problem of singular stiffness matrix and local instability in wrinkling zones. Its convergence rate strongly depends on the mass matrix formulation. In this paper, some expressions for masses are tested and their efficiency are compared in a case of inflatable lifejacket.
\end{abstract}

Key words: Form-finding / dynamic relaxation / convergence study / inflatable lifejacket

\section{Introduction}

La conception des gilets de sauvetage gonflables suit actuellement un processus artisanal de prototypage. Cependant, l'essor que connait actuellement le marché de la plaisance conduit les industriels à revoir constamment leur gamme de gilets de sauvetage, ce qui demande une meilleure réactivité en matière de création de nouveaux modèles. Dans ce contexte, la simulation numérique offre une aide très appréciable. Les méthodes numériques de recherche de forme permettent d'analyser le comportement des structures souples et d'en faciliter le dimensionnement. En particulier, de nombreux développements ont été réalisés concernant les grandes structures architecturales en toiles tendues ou gonflables $[1,2]$. Un gilet de sauvetage gonflable est constitué de deux peaux minces en textile technique soudées sur leur contour. Cette forme initialement plane (pliée dans un harnais) se déploie automatiquement lorsque le porteur tombe à la mer. La

a Auteur pour correspondance : Troufflard@univ-ubs.fr dynamique du déploiement et donc l'influence du pliage ne font pas l'objet de cette étude.

Les aspects indispensables à analyser pour la conception d'un gilet de sauvetage sont le volume de gaz qu'il peut contenir et sa forme une fois gonflé. Le volume est important car il détermine la force de remontée fournie à l'utilisateur. Cette force indique la catégorie du gilet visà-vis des normes en vigueur. La forme gonflée du gilet a quant à elle un impact sur l'ergonomie et les contraintes d'utilisation qui sont la liberté de mouvement et la capacité respiratoire du porteur. Enfin, des plis apparaissent dans certaines zones de la forme gonflée. La localisation de ces zones est importante à maîtriser car elle a également un impact sur le confort d'utilisation. La simulation numérique doit permettre de renseigner sur ces trois points essentiels. Son utilisation doit de plus être la plus simple possible car elle est destinée à des personnes non initiées au calcul par éléments-finis. Il est donc nécessaire de mettre en place une simulation avec peu de paramètres d'entrée qui soit à la fois fiable et rapide. 
Cette étude numérique par éléments finis porte sur les structures gonflables à peaux minces. Le grand déséquilibre entre leur forme initiale et le chargement de pression engendre de grands déplacements et des mouvements de corps rigide. Les méthodes de calcul statique classiques sont alors inefficaces à cause de la singularité de la matrice de raideur. Les deux sources de singularité sont l'absence de rigidité en flexion des membranes et la formation de plis dans certaines zones. Ce problème de singularité est à l'origine du développement des méthodes numériques de recherche de forme dont les plus courantes sont la méthode des densités de force [3] et la méthode de relaxation dynamique [4]. La méthode de relaxation dynamique consiste à transformer un problème statique indéterminé en problème de dynamique par l'introduction d'une force d'inertie et d'un amortissement visqueux articiels. En recherche de forme, un amortissement cinétique est souvent préféré à un amortissement visqueux. En effet, il est très difficile de se placer dans un régime d'amortissement critique à cause des grandes variations de raideur de structure. La méthode de relaxation dynamique avec amortissement cinétique [5], largement appliquée en recherche de forme [6], est une méthode classique et très flexible utilisant à la fois la méthode des éléments-finis pour la discrétisation spatiale et les différences finies pour la discrétisation temporelle du problème de dynamique [7]. L'utilisation d'une méthode dynamique permet de supprimer les problèmes de matrice de raideur singulière. Le mécanisme de dissipation de l'énergie cinétique conduit efficacement à la minimisation de l'énergie potentielle du système. Cependant, la rapidité de convergence de la méthode est fortement liée à la matrice masse qui doit être judicieusement choisie afin de réduire les temps de calcul. Ces derniers sont quant à eux liés à la taille des éléments qui doit être nécessairement petite pour prédire correctement les zones de plis.

Dans cette étude, nous étudions plus particulièrement l'influence de l'expression de la matrice masse sur la convergence. Trois expressions de cette matrice masse sont alors comparées, une forme physique classique, celle proposée par Han et al. [8] et une version modifiée de cette dernière. Dans une première partie, la méthode de relaxation dynamique est résumée. Puis, les différentes formulations des masses sont établies. Enfin, une comparaison des performances de chacune des méthodes est menée sur un cas industriel de gilet de sauvetage gonflable.

\section{Méthode de relaxation dynamique}

\subsection{Résolution du problème de dynamique}

Le problème de dynamique à résoudre est celui défini par la seconde loi de Newton :

$$
F_{\text {acc }}+F_{\text {int }}=F_{\text {ext }}
$$

avec $F_{\text {acc }}, F_{\text {int }}$ et $F_{\text {ext }}$ respectivement les forces d'accélération, internes et externes. Le chargement extérieur est modélisé par une pression suiveuse uniforme.
Après discrétisation par la méthode des éléments finis, l'expression (1) devient :

$$
[M] \ddot{X}+R(X)=0
$$

avec $[M]$ la matrice masse diagonale, $\ddot{X}$ et $X$ respectivement le vecteur accélération et position aux nouds et $R(X)$ le résidu des forces internes et externes dépendant de la position des nouds.

La méthode des différences finies centrées (DFC) est couramment utilisée pour intégrer temporellement les équations de la dynamique. Elle conduit aux approximations suivantes :

$$
\begin{aligned}
\ddot{X}_{n} & =-[M]^{-1} R\left(X_{n}\right) \\
\dot{X}_{n+\frac{1}{2}} & =\dot{X}_{n-\frac{1}{2}}+\Delta t \ddot{X}_{n} \\
X_{n+1} & =X_{n}+\Delta t \dot{X}_{n+\frac{1}{2}}
\end{aligned}
$$

avec $\Delta t$ le pas de temps. Le schéma DFC est typiquement utilisé en recherche de forme par relaxation dynamique. Dans cet article, le schéma amortissant de TchamwaWielgosz (TW) [9] est également testé dans le cas d'une matrice de masse physique. Le schéma TW est habituellement utilisé pour améliorer la réponse numérique de phénomènes de dynamique rapide en filtrant les hautes fréquences de vibrations (ondes numériques dues aux différentes discrétisations spatiale et temporelle). L'intensité de cette filtration est ajustée via un paramètre $\phi[10]$. Dans le cas transitoire, une valeur de $\phi$ trop élevée conduit à une filtration excessive et donc à une disparition de fréquences physiques. Dans nos applications, le paramètre $\phi$ a pour effet de stabiliser la structure et améliore ainsi la convergence (seul l'état statique final est en effet recherché). Le paramètre $\phi$ peut donc prendre n'importe quelle valeur supérieure ou égale à 1 . Pour ce schéma, les vitesses et positions nodales sont calculées par :

$$
\begin{aligned}
\ddot{X}_{n} & =-[M]^{-1} R\left(X_{n}\right) \\
\dot{X}_{n+1} & =\dot{X}_{n}+\Delta t \ddot{X}_{n} \\
X_{n+1} & =X_{n}+\Delta t \dot{X}_{n}+\phi \Delta t^{2} \ddot{X}_{n}
\end{aligned}
$$

\subsection{Amortissement cinétique}

$\mathrm{Au}$ cours du processus dynamique, l'énergie cinétique totale de la structure est contrôlée à chaque pas de temps. Pour chaque pic d'énergie cinétique rencontré, les vitesses nodales sont remises à 0 . Ce traitement est répété jusqu'à ce qu'un critère d'arrêt soit satisfait (soit sur le résidu des forces, soit sur les déplacements résiduels). La discrétisation temporelle conduit à une erreur sur le temps $t^{*}$ où un pic d'énergie cinétique apparaît. Il est donc nécessaire de recalculer les positions nodales à $t^{*}$ via un pas de temps correctif $\Delta t^{*}[6]$. Dans le cas du schéma TW, les vitesses et les positions sont calculées au même instant. Pour les énergies cinétiques $E c_{n-1}, E c_{n}$ et $E c_{n+1}$ respectivement aux temps $t-1, t$ et $t+1$, nous obtenons :

$$
\Delta t^{*}=\Delta t \frac{E_{n-1}-E_{n+1}}{2\left(E_{n-1}-2 E_{n}+E_{n+1}\right)}
$$


Le pas de temps $\Delta t^{*}$ permet de calculer les positions nodales $X_{n}{ }^{*}$ au pic d'énergie cinétique par rapport aux positions nodales $X_{n}$ à $t$. Si le temps $t^{*}$ est inférieur à $t$, les relations suivantes donnent les positions corrigées suivant le schéma temporel :

$$
\begin{aligned}
\text { DFC : } X_{n}{ }^{*}= & X_{n}+\Delta t^{*} \dot{X}_{n-\frac{1}{2}} \\
T W: X_{n}{ }^{*}= & X_{n}+\Delta t^{*} \dot{X}_{n-1} \\
& +\phi\left(2 \Delta t \Delta t^{*}+\Delta t^{* 2}\right) \ddot{X}_{n-1}
\end{aligned}
$$

Si le temps $t^{*}$ est supérieur à $t$, cela équivaut à remplacer $\Delta t$ par $\Delta t^{*}$ dans les expressions (3) ou (4).

\subsection{Formulation des forces internes}

Un élément-fini de membrane triangulaire à trois nœuds et 3 degrés de liberté par nœud est utilisé pour la discrétisation spatiale. Le matériau est élastique isotrope et nous faisons l'hypothèse des contraintes planes. La puissance virtuelle des efforts internes s'écrit :

$$
P_{i n t}^{*}=\int_{\mathcal{D}} \sigma^{i j} \stackrel{*}{V_{i \mid j}} \mathrm{~d} v
$$

avec $\sigma^{i j}$ le tenseur des contraintes et $V_{i \mid j}^{*}$ le tenseur des vitesses virtuelles de déformation. Une base naturelle $g_{i}$ est associée à chaque élément. Les composantes locales du tenseur des déformations d'Euler-Almansi sont données à partir du tenseur métrique initial $G_{i j}^{0}$ et actuel $G_{i j}$ dans la base naturelle par :

$$
\epsilon_{i j}=\frac{1}{2}\left(G_{i j}-G_{i j}^{0}\right)
$$

Le tenseur des contraintes est calculé par :

$$
\sigma^{i j}=E_{n k}^{i}{ }^{l} \epsilon_{m l} G^{k m} G^{j n}
$$

avec $E^{i}{ }_{n k}{ }^{l}$ le tenseur des rigidités du matériau élastique et $\epsilon_{m l}$ le tenseur des déformations et $G^{i j}$ le tenseur métrique dans la base duale. Le tenseur des vitesses virtuelles de déformation s'exprime par :

$$
V_{i \mid j}^{*}=V_{, j}^{*} \cdot g_{i}
$$

avec $V_{, j}^{*}$ le gradient des vitesses virtuelles et $g_{i}$ les vecteurs de la base naturelle. L'expression (10) s'écrit sur un élément de référence :

$$
V_{i \mid j}^{*}=\left(V^{k}\right) \varphi_{l, i} \varphi_{k, j}\left(X^{l}\right)
$$

avec $\varphi_{i, j}$ la dérivée de la fonction d'interpolation au nœud $i$ par rapport à la composante spatiale $j,\left(X^{l}\right)$ le vecteur position du nœud $l$ et $\left(V^{k}\right)$ les vitesses virtuelles. L'expression des forces internes au nœud $k$ est obtenue en sommant sur chaque élément $e$ incluant ce nœud $k$ :

$$
F_{k}=\sum_{e} S_{e} h_{e} \sigma^{i j} \varphi_{l, i} \varphi_{k, j}\left(X^{l}\right)
$$

avec $S_{e}$ et $h_{e}$ respectivement la surface et l'épaisseur de l'élément $e$.

\subsection{Critère d'arrêt}

Il est classique en relaxation dynamique d'arrêter le calcul soit par un critère sur le résidu des forces, soit sur les déplacements ou bien encore une combinaison des deux. Cela traduit la tolérance souhaitée sur l'équilibre statique. Nous choisissons dans cette étude d'utiliser un critère sur les déplacements. Afin que ce critère s'adapte au cas étudié, il faut prendre en compte les données physiques du problème, notamment les caractéristiques élastiques du matériau et le chargement de pression. De plus, le pas de temps a une influence sur la distance parcourue par les nœuds au cours d'un incrément. À partir de ces données, le critère en déplacement suivant est proposé pour chaque noud $i$ :

$$
U_{\max }^{i}=a \frac{p}{h \rho_{i}} \Delta t^{2}
$$

où $p$ est la pression de gonflage, $\rho_{i}$ la masse volumique associée au nœud $i$ et $a$ un paramètre permettant d'ajuster ce critère.

Ce critère peut être interprété comme l'amplitude d'oscillation d'un système non amorti autour de sa position d'équilibre. Quel que soit le type de matrice masse utilisé, les caractéristiques du matériau seront prises en compte par la relation de stabilité du schéma explicite. Dans le cas des masses physiques, $\rho_{i}$ est fixe et le pas de temps est restreint par le pas de temps critique. Pour l'élément triangle choisi, on a la relation suivante :

$$
\Delta t \leq \min _{e}\left(\frac{2 S^{e}}{L_{\max }^{e}} \sqrt{\frac{\rho^{e}\left(1-\nu^{2}\right)}{E}}\right)
$$

où $L_{\max }^{e}$ est la longueur de la plus grande arête, $\rho^{e}$ la masse volumique et $S^{e}$ la surface de l'élément $e$.

\section{Formulation de la matrice masse}

L'analyse de convergence réalisée par [11] montre qu'un choix approprié de la matrice masse conduit à une convergence optimale de la méthode de relaxation dynamique. Cette matrice masse est obtenue à partir de la condition CFL [12] qui donne la valeur du pas de temps maximum. Pour un élément $e$, cette limite sur le pas de temps peut être exprimée par :

$$
\Delta t \leq \frac{2}{\omega_{\max }^{e}}
$$

où $\omega_{\max }^{e}$ est la fréquence maximum de l'équation (2) pour l'élément $e$. Cette fréquence $\omega_{\max }^{e}$ est obtenue par :

$$
\omega_{\max }^{e}=\max \sqrt{A^{e}}
$$

où $A^{e}$ représente les valeurs propres de la matrice $\left([M]^{e^{-1}}[K]^{e}\right),[M]^{e}$ et $[K]^{e}$ étant respectivement la matrice masse et de raideur pour l'élément $e$. Si on exprime $[K]^{e}$ dans ses directions principales et en prenant 
$[M]^{e}$ diagonale, on peut réécrire l'expression (16) sous la forme :

$$
\omega_{\max }^{e}=\max \sqrt{\frac{k_{j j}^{e}}{m_{j j}^{e}}}
$$

où $m_{j j}^{e}$ et $k_{j j}^{e}$ sont respectivement les termes des matrices masse et de raideur diagonalisées. En injectant (17) dans (15), on obtient la masse optimale pour l'élément $e$ :

$$
m^{e} \geq \frac{\Delta t^{2}}{4} k_{\max }^{e}
$$

où $k_{\max }^{e}$ est la valeur maximum parmi les termes de $[K]^{e}$ diagonalisée. La masse pour un nœud $i$ est donnée par :

$$
m_{i} \geq \sum_{e} m^{e}=\frac{\Delta t^{2}}{4} \sum_{e} k_{\max }^{e}
$$

En pratique, le terme $k_{\max }^{e}$ n'est pas calculé de manière exacte. Les larges déplacements engendrés par les efforts extérieurs conduisent à une grande variation de la raideur aux éléments et donc des valeurs propres de $[K]^{e}$. Un calcul exact de ces valeurs propres à chaque incrément serait trop coûteux en temps de calcul. Seule une évaluation de l'expression $k_{\max }^{e}$ est alors donnée. Une expression similaire des termes $m_{i}$ de la matrice masse, dans le cas monodimensionnel, est obtenue par [13] :

$$
m_{i}=\lambda \frac{\Delta t^{2}}{2} k_{i \max }
$$

avec $k_{i \text { max }}$ la raideur maximale approximée au nœud $i$ et $\lambda$ un paramètre de convergence constant pour toute la structure. Moins l'évaluation de $k_{i \max }$ est précise, plus $\lambda$ sera grand afin de compenser l'erreur commise. Le pas de temps $\Delta t$ est arbitraire pour la recherche de forme et typiquement égal à 1 .

Le terme de raideur $k_{i \max }$ peut être décomposé en une partie relative au matériau et une partie géométrique dépendante de l'état de contrainte dans la membrane :

$$
k_{i \max }=\sum_{e}\left(k_{\mathrm{mat}}^{e}+k_{\mathrm{geo}}^{e}\right)
$$

Pour un élément membrane à trois nœuds, l'expression suivante est proposée par [8] :

$$
k_{i \max }=\sum_{e} \frac{h}{4 S_{0}^{e}}\left(\frac{E}{1-\nu^{2}}+\sigma_{x}+\sigma_{y}+\sigma_{x y}\right)
$$

avec $S_{0}^{e}$ la surface initiale de l'élément $e$ et $h$ l'épaisseur de la membrane. $\sigma_{x}, \sigma_{y}$ et $\sigma_{x y}$ sont les composantes du tenseur des contraintes. $E$ et $\nu$ sont les coefficients d'une loi élastique isotrope.

Cette expression permet une évaluation assez précise de la raideur environnant chaque nœud mais on s'aperçoit que le terme obtenu en injectant (22) dans (20) n'a pas la dimension d'une masse. Pour éviter ce problème d'homogénéité des dimensions, la forme modifiée suivante est proposée :

$$
k_{i \max }=\sum_{e} \frac{h}{4}\left(\frac{E}{1-\nu^{2}}+\sigma_{x}+\sigma_{y}+\sigma_{x y}\right)
$$

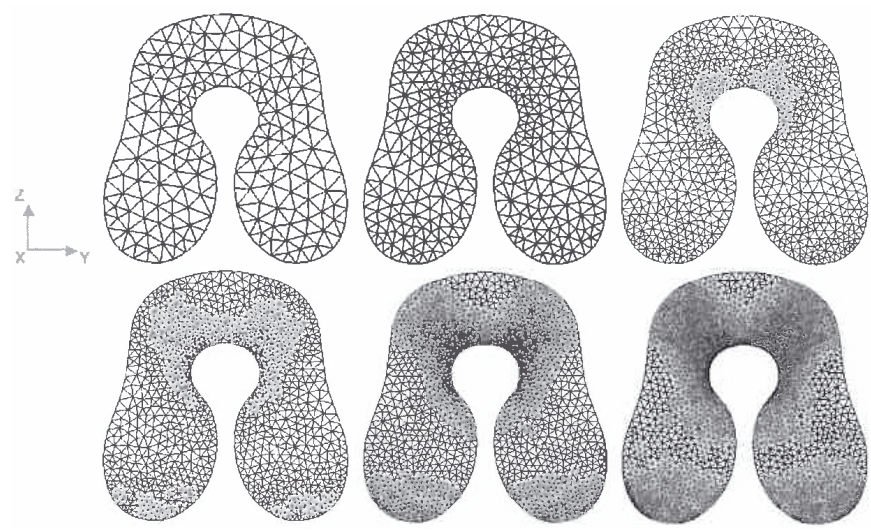

Fig. 1. Maillages de gilet $150 \mathrm{~N}$ (de gauche à droite) : 1053 ddl (698 éléments), 1971 ddl (1310 éléments), 4590 ddl (3056 éléments), 7611 ddl (5070 éléments), 11349 ddl (7562 éléments), 16506 ddl (11000 éléments).

L'expression (20) offre une bonne approximation des masses pour avoir une convergence optimale. De ce fait, la matrice physique classique des masses a peu d'intérêt en terme de rapidité de convergence. Cependant, les masses physiques sont introduites ici comme élément de comparaison en terme de performance. Toujours dans le cas d'éléments membrane triangulaires à trois nœuds, la masse physique $m_{i}$ au nœud $i$ est obtenue en sommant sur chaque élément $e$ incluant le nœud $i$ à l'aide de l'expression suivante :

$$
m_{i}=\frac{1}{3} \sum_{e} \rho S_{0}^{e} h
$$

avec $\rho$ la masse volumique des peaux de l'enveloppe gonflable.

\section{Application au gilet de sauvetage gonflable}

Dans cette partie, les différentes formulations de masse sont testées sur un cas de gilet de sauvetage gonflable de catégorie $150 \mathrm{~N}$. Une application à un gilet $275 \mathrm{~N}$ est également présentée.

Les deux peaux du gilet sont modélisées entièrement, sans prendre en compte les symétries de la géométrie. D'une part, les nouvelles formes conçues en bureau d'étude pourront présenter des dissymétries. D'autre part, cela permet de limiter les conditions de blocage qui peuvent influencer la localisation des zones de plis. La structure est donc gonflée, par une pression uniforme, sans condition limite de blocage. La méthode numérique en dynamique permet de le faire sans singularité dans la résolution du problème. Les données pour ce calcul sont les suivantes :

- module de Young : $E=125 \mathrm{MPa}$;

- coefficient de Poisson : $\nu=0,41$;

- pression de gonflage : $p=150$ mbar;

- épaisseur de membrane : $e p=0,27 \mathrm{~mm}$. 

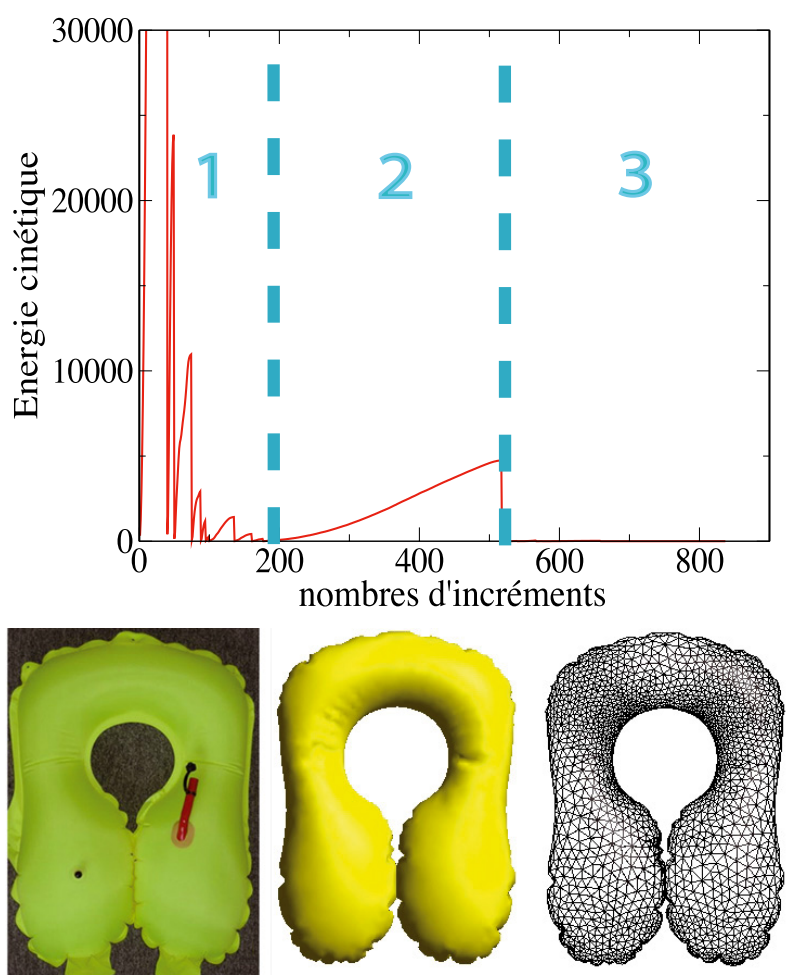

Fig. 2. (a) Évolution de l'énergie cinétique en fonction du nombre d'incréments. Visualisation des trois phases : 1- amortissement du déséquilibre initial, 2- mouvement vers le plan $(Y=0)$, 3- stabilisation finale, (b) gilet de sauvetage gonflable $150 \mathrm{~N}$ gonflé à 150 mbar (à gauche : gilet réel, à droite : résultat de simulation lissé et maillage déformé (11349 ddl, 7562 éléments)).

Les maillages testés sont présentés sur la figure 1. La forme plane est initialement dans le plan $(X=0)$. Au voisinage de la position d'équilibre du gilet gonflé, les deux parties inférieures sont en contact. L'interpénétration de ces zones est évitée par l'application d'une force, aux nouds franchissant le plan $(Y=0)$, équivalente à une méthode de pénalisation. Le déroulement classique du calcul par relaxation dynamique est illustré sur la figure 2a montrant la dissipation progressive de l'énergie cinétique du système. On distingue trois phases sur cette courbe. Dans la première phase, de hauts niveaux d'énergie cinétique sont atteints découlant du fort déséquilibre entre la forme initiale plane du gilet et son chargement de pression. La seconde phase traduit le mouvement des parties inférieures du gilet vers le plan $(Y=0)$. Dans la troisième phase, on observe la stabilisation finale de la géométrie. La géométrie est considérée stable lorsqu'il y a eu respect du critère d'arrêt (13) pendant cinq incréments consécutifs. La forme gonflée du gilet $150 \mathrm{~N}$ réel et un exemple de résultat numérique sont montrés sur la figure $2 \mathrm{~b}$. Le volume réel du gilet est d'environ $21000 \mathrm{~cm}^{3}$. Lors des diverses simulations réalisées, nous trouvons un volume typiquement compris entre 19838 (maillage à 698 éléments) et 20593 (maillage à 11000 éléments) soit un écart respectivement de 5,53\% et $1,94 \%$. Numériquement, la localisation des zones de

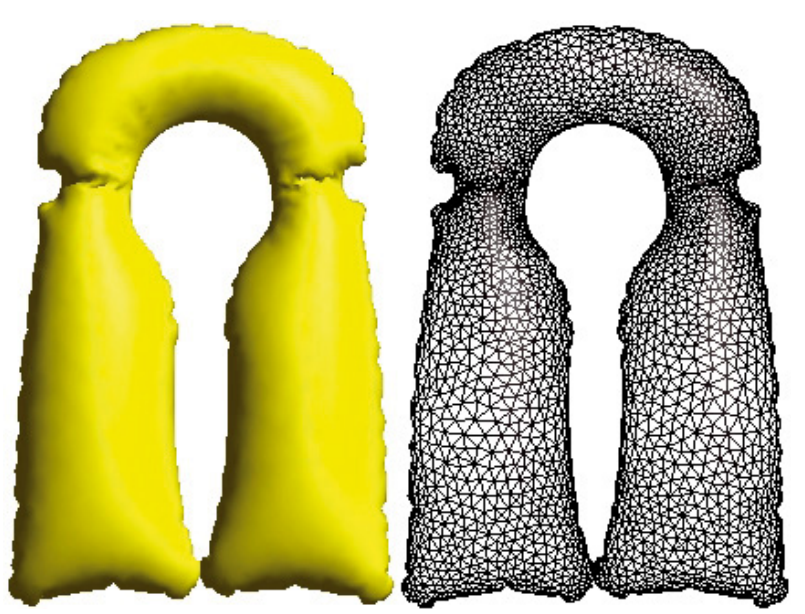

Fig. 3. Gilet de sauvetage gonflable $275 \mathrm{~N}$ gonflé à $150 \mathrm{mbar}$ (à gauche : résultat de simulation lissé, à droite : maillage déformé (15 465 ddl, 10306 éléments)).

plis devient réellement possible à partir du maillage à 3056 éléments, soit une densité de maillage minimum d'environ un nœud par centimètre. En ce qui concerne le temps de calcul, l'objectif visé est d'environ 30 min pour une utilisation efficace en bureau d'études. Cependant, lors de son utilisation en industrie, les paramètres de calcul optimum ne seront pas toujours appliqués. L'un des buts de ce travail est de réduire la sensibilité du temps de calcul aux paramètres d'entrée. C'est pourquoi l'influence du paramètre $\lambda$ de l'expression (20) a été testée pour les différentes finesses de maillage de la figure 1. Les résultats pour les expressions (22) et (23) des masses sont regroupés respectivement dans les tableaux 1 et 2 . Le tableau 1 montre également des résultats pour une matrice de masse physique avec les schémas DFC et TW.

Tout d'abord, on constate que l'expression (22) apporte une bonne amélioration de la convergence par rapport aux masses physiques. Le schéma TW permet une légère amélioration par rapport à la DFC pour les masses physiques mais nécessite de déterminer la valeur optimum du paramètre $\phi$. Le paramètre $\lambda$ dans le cas de la formulation (22) est très sensible à la finesse du maillage. L'expression modifiée (23) apporte à la fois une réduction de cette sensibilité et une amélioration de la vitesse de convergence, notamment pour les maillages les plus denses. Ceci a un intérêt concret dans son application en bureau d'étude. L'utilisateur est ainsi assuré de disposer d'une simulation efficace quelle que soit la finesse du maillage sans avoir à rechercher le paramètre $\lambda$ optimum. Une valeur de $\lambda$ fixée à trois suffit pour mener les calculs. Le résultat numérique de la figure $2 \mathrm{~b}$ a été obtenu en 16 min sur une station de calcul (CPU 3,20 GHz RAM 2 Mo). Dans les mêmes conditions, un gilet de sauvetage $275 \mathrm{~N}$, modélisé par 10306 éléments et montré sur la figure 3, a été obtenu en 28 min. Pour ce gilet, le volume numérique de $35774 \mathrm{~cm}^{3}$ présente un écart de $2,21 \%$ par rapport au volume réel de $35000 \mathrm{~cm}^{3}$. 
Tableau 1. Influence de $\lambda$ sur le nombre d'incréments de résolution (masses calculées selon expression (22)), résultats avec matrice masse physique en utilisant le schéma DFC, et résultats avec matrice masse physique en utilisant le schéma TW pour différentes valeurs du paramètre $\phi$.

\begin{tabular}{ccllccccccc}
\hline Nb ddl & $\lambda:$ & 100 & 400 & 800 & 1000 & 2000 & 3000 & 4000 & DFC & TW \\
\hline 1053 & nb incr : & div & div & div & div & div & 290 & 335 & 438 & $361(\phi=1,01)$ \\
1971 & nb incr : & div & div & div & div & 524 & 643 & 682 & 752 & $659(\phi=1,06)$ \\
4590 & nb incr : & div & div & div & 1261 & 1909 & 2382 & 2893 & 1432 & $1386(\phi=1,01)$ \\
7611 & nb incr : & div & div & 1860 & 2396 & 3217 & 3790 & 4651 & 2005 & $2185(\phi=1,01)$ \\
11349 & nb incr : & div & div & 3058 & 3234 & 4903 & 5763 & 6003 & 3288 & $2781(\phi=1,02)$ \\
16506 & nb incr : & div & 3165 & 4245 & 4871 & 7376 & 8219 & 10005 & 3453 & $3003(\phi=1,02)$ \\
\hline \multicolumn{10}{c}{ div $=$ divergence, critère d'arrêt expression $(13)$ pour $a=0,5$} \\
\hline
\end{tabular}

Tableau 2. Influence de $\lambda$ sur le nombre d'incréments de résolution (masses calculées selon expression (23)).

\begin{tabular}{cccccccc}
\hline Nb ddl & $\lambda:$ & 2. & 2.5 & 3 & 4 & 5 & 6 \\
\hline 1053 & nb incr : & div & 221 & 240 & 274 & 307 & 335 \\
1971 & nb incr : & div & 325 & 337 & 393 & 438 & 479 \\
4590 & nb incr : & div & div & 619 & 734 & 924 & 1017 \\
7611 & nb incr : & div & 694 & 941 & 1086 & 1172 & 1346 \\
11349 & nb incr : & div & 839 & 1359 & 1255 & 1301 & 1582 \\
16506 & nb incr : & div & 1367 & 1475 & 1958 & 1912 & 2033 \\
\hline \multicolumn{7}{l}{ div $=$ divergence, critère d'arrêt expression $(13)$ pour $a=0,5$} \\
\hline
\end{tabular}

\section{Conclusion et perspectives}

La méthode de relaxation dynamique permet d'obtenir rapidement la forme finale d'une structure gonflable, malgré les instabilités inhérantes à ce type d'équilibre. Dans cette étude, nous nous sommes plus particulièrement intéressés à l'influence de la matrice masse sur la convergence. Pour ceci, une comparaison entre plusieurs formulations de matrice masse a été réalisée. L'expression de Han and Lee a été modifiée pour améliorer la rapidité de convergence et minimiser l'influence de la finesse de maillage. Grâce à ces améliorations, cette méthode numérique est aujourd'hui utilisée en industrie pour le dimensionnement des gilets de sauvetage gonflables.

Notons également que malgré son intérêt, la relaxation dynamique avec amortissement cinétique ne peut s'employer en l'état, pour des comportements matériels dépendant du trajet de chargement tels que la plasticité.

Enfin, la méthode présentée doit pouvoir a priori se généraliser à d'autres types d'éléments-finis.

Ainsi, les travaux en cours visent à étendre la méthode à des découpages quadrangulaires et des interpolations plus riches. Les premiers résultats obtenus sont encourageants car la méthode converge également pour des interpolations quadratiques.

Remerciements. Cette étude a été financée par la région Bretagne et en collaboration avec la société Plastimo. Les auteurs remercient en particulier Mr Bernard Geneau pour ses conseils.

\section{Références}

[1] D.S. Wakefield, Engineering analysis of tension structures: theory and practice, Eng. Struct. 21 (1999) 680-690
[2] W.J. Lewis, Tension Structures, Form and Behaviour, in : T. Telford (éd.), 2003

[3] H.J. Schek, The force densities method for form-finding and computation of general networks, Comput. Methods Appl. Mech. Eng. 3 (1974) 115-134

[4] A.S. Day, An introduction to dynamic relaxation. The engineer, technical contributors section, 1965, pp. 220221

[5] P.A. Cundall, Explicit finite-difference methods in geomechanics, In Proc. E.F. Conf. on Numerical Methods in Geomechanics, Blacksburg, June 1976

[6] M.R. Barnes, Form-finding and analysis of prestressed nets and membranes, Comput. Struct. 30 (1988) 685-695

[7] W.J. Lewis, The efficiency of numerical methods for the analysis of prestressed nets and pin jointed frame structures, Comput. Struct. 33 (1989) 791-800

[8] S.-E. Han, K.-S. Lee, A study of the stabilizing process of unstable structures by dynamic relaxation method, Comput. Struct. 81 (2003) 1677-1688

[9] B. Tchamwa, Contributions à l'étude des méthodes d'intégration directe explicites en dynamique non linéaire des structures Ph.D. thesis, École Centrale de Nantes, 1997

[10] G. Rio, A. Soive, V. Grolleau, Comparative study of numerical explicit time integration algorithms, Adv. Eng. Softw. 36 (2005) 252-265

[11] P. Underwood, Dynamic Relaxation in: Computational Methods for Transient Analysis, in: T. Belytshko, T.J.R. Hugues (éd.), 1983, pp. 245-265

[12] R. Courant, K. Friedrichs, H. Lewy, On the partial difference equations of mathematical physics, Mathematische Annalen 100 (1928) 32-74

[13] M.R. Barnes, Form-finding and analysis of tension structures by dynamic relaxation, Int. J. Space Struct. 14 (1999) 89-104 